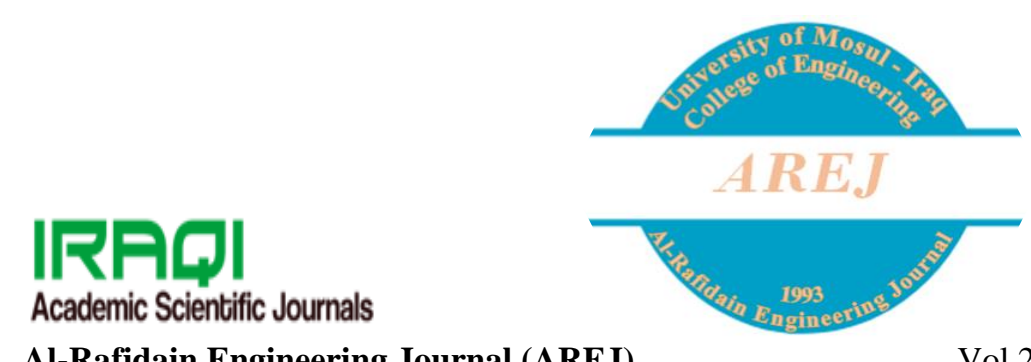

\title{
Beam Tracking Channel for Millimeter-Wave Communication System Using Least Mean Square Algorithm
}

\author{
Ban A. Asi* \\ ban.enp1@student.uomosul.edu.iq \\ Farhad E. Mohmood** \\ farhad.m@uomosul.edu.iq \\ * Computer Engineering Department, Collage of Engineering, University of Mosul \\ ** Electrical Engineering Department, Collage of Engineering, University of Mosul
}

Received: $13 / 12 / 2020$

Accepted: 14/2/2021

\begin{abstract}
Millimeter-wave (mmwave)is an attractive option for high data rate applications in the $5 G$ wireless communication that requires proper beamforming, channel tracking, and channel change. Adaptive beams are formed by relying on adaptive algorithms. In this paper, we study, analyze, and compare the performance of the least mean square algorithm (LMS) and normalized least mean square (NLMS) for tracking channel status and transmit array beam. When using LMS algorithms and natural NLMS algorithms, an adaptive filter usually results in a trade-off between convergence velocity and adaptive accuracy. The results showed that the LMS algorithm is one of the simplest types of algorithm but it needs a large step size to obtain faster system convergence and stability. NLMS algorithm is a special application for the LMS algorithm, in which NLMS algorithm takes into account the change in the signal level when applying the filter and specifies the normal step size parameter $\mu$. this leads to stability as well as rapid convergent adaptation of the algorithm.
\end{abstract}

Keywords:

5G, Massive multi input multi output(Massive MIMO), Millimeter-wave (mmwave), Adaptive filter, Least mean square(LMS), Normalized least mean square(NLMS)..

This is an open access article under the CC BY 4.0 license (http://creativecommons.org/licenses/by/4.0/). https://rengj.mosuljournals.com

\section{INTRODUCTION}

Recently, Massive multiple-inputs multiple-outputs (MIMO) frameworks are used in the fifth generation of mobile communication systems $(5 \mathrm{G})$ to enhance system efficiency and to reduce interference from multiple users as a large number of antennas are provided to the base station[1]. With the increasing demand for high spectral efficiency and high bandwidth, millimeter-wave (mmWave) technology is adopted with massive MIMO technology in 5G. mmWave's short-wavelength helps shorten the spacing of antennas. Beamforming is a major enabler of mmWave communication[2][3]. The beams must be synchronized between the base station (BS) and the associate user equipment (UEs) due to the high directivity when using beam modulation[4]. To get hold advantage of beamforming technology, in mmWave range, accurate angle tracking is required. As the wireless user is moving in different directions, it is important to monitor the channel gain and the beam angle simultaneously. As the angle of the beam follows a non-linear function, non-linear tracking systems need to be used [5].

One way to track the beam is via the Ray tracing system. However, these methods involve a high computational complexity. Another way to track the beam is through some filters. These filters can be divided into two types: an adaptive filter and a fixed filter. The static filter is helpful when the signal and channel parameters are known. On the other hand, adaptive filters are useful when the dynamics of a signal or channel are unpredictable and change over time. An adaptive filter is a filter that works under the control of some algorithms such as the least mean square (LMS), and the recursive least square (RLS) [6].

The authors in [7] show developed an adaptive noise canceling device by applying LMS and NLMS algorithms. Because LMS has a fixed 
step size, it is not suitable for working in an unstable environment. But for NLMS, is suitable for working in an unstable environment as well as a static environment. The research in [8] used algorithms Least mean square (LMS), Simple matrix inversion (SMI), Recursive least mean square(RLS). It was observed that with an increase in the number of elements, an improvement in beamwidth and reduced interference between users could be obtained The work in [9] proposed the Kalman beam tracking algorithm as a solution to reduce beam training overhead. An interesting effect was the size of the matrix. At the same SNR, the array must be chosen to be large enough for optimal performance .The research in [6] studied the effect of step size and the number of iterations on filter performance such as squared error, estimation accuracy, etc. The decrease in step size reduces steady-state error but at the same time increases convergence time. in the mmWave mobile communication case, The authors in [10] show derived the LMS algorithm and the BiILMS extension algorithm are derived from the steeper main algorithm. The numerical results show that (LM)S and (BiLMS) rapidly converge with the best performance for increased SNR. On the other hand, with an increasing SNR, the (RLS) algorithm exhibits a relatively slow convergence.

In this paper, the algorithms of (LMS, NLMS) to track the channel in communications mmWave were studied and analyzed. The results show that the algorithm of LMS is less complex compared to the algorithm of (NLMS) it reduces the difference between the desired signal and the actual signal of an unknown system where the MSE standard is used, and the time of implementation of the algorithm.

The remainder of this paper is organized as follows in Section II, the system model is presented with beamforming, in Section III, the algorithms (LMS, NLMS) are derived, in Section IV, we produced the numerical result, finally, some conclusions are present in Section V.

\section{SYSTEM MODEL}

In this section, channel pattern and beamforming model are discussed along with channel monitoring.

\subsection{Model Of Time-Varying mmwave Channel Model}

Here, because of its directional nature the geometric time-varying channel model can be used to model the mmwave channel. The time index is $k$ and the channel can be represented as:[10]
$H_{k}=\sum_{l=1}^{L} \alpha_{k, l} a_{R}\left(\theta_{k, l}\right) a_{T}\left(\Phi_{k, l}\right)^{T}$

where $L$ is the wireless path which ranges from 1 to $L, \alpha_{k, l}$ is the complex path gain, $\theta_{k, l}$ and $\Phi_{k, l}$ are the angle of departure (AOD) and angle of arrival (AOA) respectively.

The response vector of the arrays $a_{R}($.$) and a_{T}($. can be expressed as follows:[9][10]

$a_{R}(\theta)=\frac{1}{\sqrt{M}}\left[1 e^{-2 j \pi \frac{d}{\lambda} \cos (\theta)} \ldots . e^{-j 2 \pi \frac{d}{\lambda}(M-1) \cos (\theta)}\right]^{T}$

(2)

$a_{T}(\Phi)=\frac{1}{\sqrt{N}}\left[1 e^{-2 j \pi \frac{d}{\lambda} \cos (\Phi)} \ldots . e^{-j 2 \pi \frac{d}{\lambda}(N-1) \cos (\Phi)}\right]^{T}$

(3)

Where $\lambda$ is the wavelength, in the uniform linear array (ULA), $d$ is the spacing of the antenna, the number of transmitter antennas is $N$. The number of receiver antennas IS $M,[.]^{T}$ is transpose of[. ].

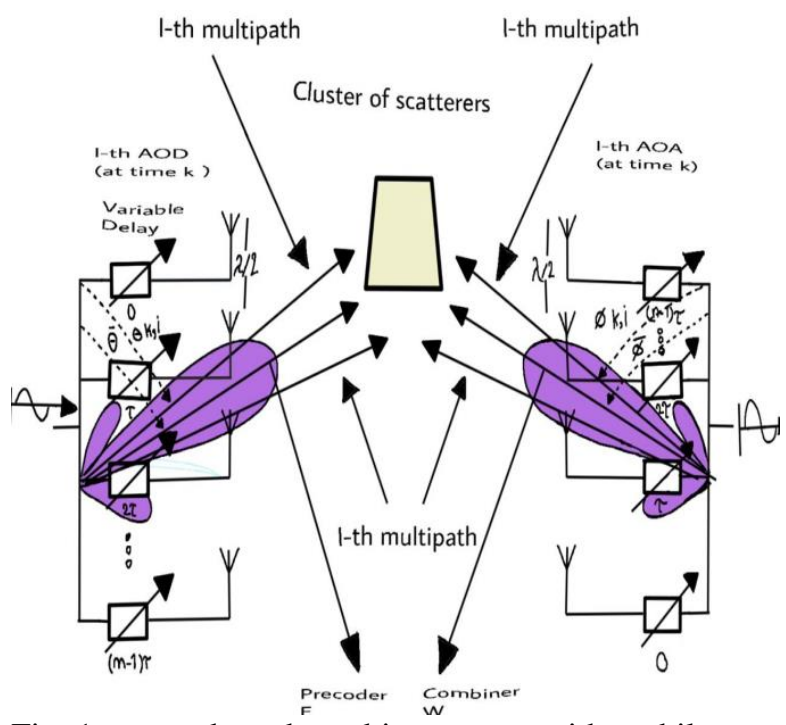

Fig. 1. precode and combiner vector with mobile mmwave communication setting and a single cluster of scattering.

To model the real channel that we want to track, we need to model the temporal evolution of the complex path gain was modeled using an automatic first-order regression approach as follow: [10]

$\alpha_{(k+1)}=\rho \alpha_{k}+u_{k}^{\alpha}$,

Where the complex of path gain vector is $\alpha_{k}$ for all the multiple paths $\alpha_{k}=$ $\left[\alpha_{k, 1} \alpha_{k, 2} \ldots \alpha_{k, L}\right]^{T}$, following a zero-mean complex Gaussian noise with covariance (1- 
$\left.\rho^{2}\right) I_{L}, I_{L}$ is the identity matrix with a size equal to $L$, the respective noise of innovation $u_{k}^{\alpha}$, the correlation of coefficient is $\rho$. In addition, the angular variation over time is modeled as a form of Gaussian noise as follows: [10]

$\theta_{(k+1)}=\theta_{k}+u_{k}^{\theta}$,

$\Phi_{(k+1)}=\Phi_{k}+u_{k}^{\Phi}$

Where:

$\theta_{k}=\left[\begin{array}{llll}\theta_{k, 1} & \theta_{k, 2} \ldots \theta_{k, L}\end{array}\right]^{T}, \Phi_{k}=$ $\left[\Phi_{k, 1} \Phi_{k, 2} \ldots \ldots \Phi_{k, L}\right]^{T}$.

Also, follow independent zero-mean complex Gaussian processes with covariance processes $\sigma_{\theta}^{2} I_{L}$ and $\sigma_{\Phi}^{2} I_{L}$ with $u_{k}^{\Phi}[9][10]$.

\subsection{The Beamforming Model}

The gain of antenna is dependent on the direction in which the signal is transmitted or the direction from which the signal is received. thus to have a large gain towards the desired direction, one has to rotate the antenna towards that angle. To overcome this problem we can use the technology beamforming. In this paper we consider a fully analog transceiver with the transmit precoder $f$ and receive combiner $w$ as in fig (1) the precoder and combiner can be expressed as: [10]

$f=\frac{1}{\sqrt{M}}\left[1 e^{-2 j \pi \frac{d}{\lambda} \cos \bar{\theta}} \ldots \ldots . e^{-j 2 \pi \frac{d}{\lambda}(M-1) \cos \bar{\theta}}\right]^{T,}$,

$w=\frac{1}{\sqrt{N}}\left[1 e^{-2 j \pi \frac{d}{\lambda} \cos \bar{\Phi}} \ldots \ldots . e^{-j 2 \pi \frac{d}{\lambda}(N-1) \cos \bar{\Phi}}\right]^{T}$

(8)

Where $\bar{\theta}$ and $\bar{\Phi}$ are the precoder and combiner vector pointing directions.

\section{TRACKING OF THE ADAPTIVE BEAM AND CHANNEL}

In this section, the algorithms (LMS), (NLMS) will be preseted to estimate unknown mmwave channel parameters AOA, AOD, and path gain.

\subsection{Least Mean Square Algorithm (LMS)}

In signal processing applications, the least mean square (LMS) adaptive algorithm commonly utilizes the gradient vector estimation from the steepest descent. To determine the optimal weight vector, an iterative process is required to update the weight vector in direction of the negative gradient vector, the mean square error value is minimized by this algorithm, It is relatively easy and does not require any matrix inversion calculation or correlation function calculation [11].

Instead of estimating the weight, we will use the (LMS) algorithm to adaptively track the states $x_{k}$, which represents the measured states of the beam directions $\theta$ and $\Phi$ and the components of the complex channel gain: the real $\alpha_{R}$ and the imaginary $\alpha_{I}$ as

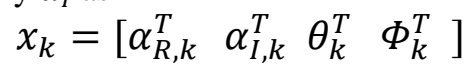

In order to track the channel and adaptive beam for the scenario of the communication, we will first consider the LMS algorithm, in the state vector $x_{k}$ since the measurement is not linear, we, therefore, (LMS) algorithm can be derived using the original algorithm of steepest descent algorithm given as [10]:

$\hat{x}_{(k+1)}=\hat{x}_{k}-\mu \nabla_{\hat{x}_{k}} J_{k^{\prime}}$

where $\hat{x}_{k}$ is the estimation of $x_{k}$, We reflect the algorithm's adaptation step-size in (9) by the diagonal matrix $\mu=\left[\begin{array}{lll}\mu_{\alpha} 1_{2 L} & \mu_{\theta} 1_{L} & \mu_{\phi} 1_{L}\end{array}\right]$, where $\mu_{\alpha}, \mu_{\theta}$ and $\mu_{\phi}$ represent the particular channel path gain, AOD, and AOA respectively, the gradient operator is $\nabla$, the mean square error at time $\mathrm{k}$ is $J_{k}$.

The estimation error is $e_{(k)}=y_{(k)}-h_{\left(\hat{x}_{k}\right)}$, have a real part $e_{R, k}$ and an imaginary part $e_{I, k}$, the MSE gave as $J_{k}=E\left\{\left\|e_{k}\right\|^{2}\right\}$ with $e_{k}=\left[e_{R, k} e_{I, k}\right]^{T}$ The costfunction wich we want minimize gave as:

Costfunction $=\operatorname{argmin}\left(J_{K}\right)$

The gradient of the MSE is given as: [10]

$\nabla_{\hat{x}_{k}} J_{k}=-2 e_{k}^{T} \frac{\partial h\left(\hat{x}_{k}\right)}{\partial \hat{x}_{k}}$

$Z_{k}=\frac{\partial h\left(\hat{x}_{k}\right)}{\partial \hat{x}_{k}}$

$\hat{x}_{(k+1)}=\hat{x}_{k}+2 \mu e_{k}^{T} Z_{k}$

\subsection{Normalized Least Mean Square Algorithm (NLMS)}

The normalized least mean square (NLMS) algorithm belongs to the adaptive algorithm gradient class that provides the solution to the slow convergence of the least mean square algorithm (LMS). In the algorithm of the normalized least mean square (NLMS), the relationship is the same for adaptively changing 
states in the (LMS) algorithm in least mean square it's difficult to select a learning rate that ensures algorithm stability. The only adjustment in (NLMS) is to normalize the input in order to solve the stated problem [12].

Normalizing the input vector provides the NLMS the ability to solve the problem by letting the step size adapt with the status of the vector:[12]

$\hat{x}_{(k+1)}=\hat{x}_{k}+\frac{\mu}{\left\|x_{k}\right\|^{2}} e_{k}^{T} Z_{k}$,

Where $\mu$ is the step size of the adaptive filter, $\left\|x_{k}\right\|^{2}$ is the normalization norm vector of $x_{k}$.

\section{NUMERICAL RESULTS AND DISCUSSION}

Depending on the program (matlab) to simulate the performance of algorithms (LMS) and (NLMS) in the mmwave beam tracking in this section. we suppose the multi-antenna system with $\mathrm{N}=16, \mathrm{M}=16$, and $\mathrm{SNR}=30 \mathrm{~dB}$, the space between antenna $\mathrm{d}=0.9 \mathrm{~cm}$, we assume multipath $\mathrm{L}=1$ for a narrow physical beam this is very likely, the time evolution of the channel underlying mmwave it's regulated by $\rho=0.995$ and $\sigma_{\theta}^{2}$ and $\sigma_{\Phi}^{2}=(0.1)^{2}$, and the vector of the precoder and combiner the arbitrary direction of $45^{\circ}$ is pointed out $\left(\bar{\theta}=\bar{\phi}=45^{\circ}\right)$. For (LMS, NLMS, RLS) for the adaptive $\mu_{\alpha}=0.1$ and $\mu_{\theta}=$ $\mu_{\Phi}=0.0001$ we use the better step-size value, and the wavelength $\lambda=0.9 \mathrm{~cm}$.

\section{RESULTS AND DISCUSSION}

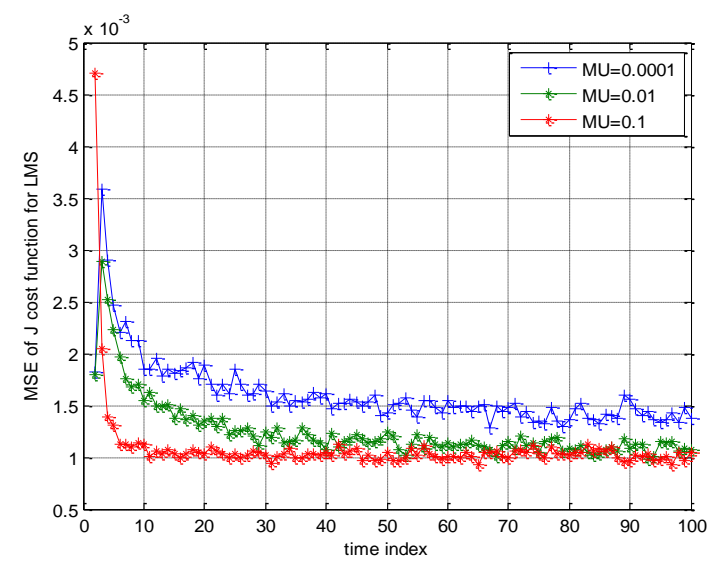

Fig. 2. comparison between cost function of the LMS algorithm for different value of $\mu$.

Fig.( 2) shows the cost function for the algorithm (LMS) for different values of the step size $\mu$, where $\mu$ is the step size parameter, and it controls on the convergence characteristics of the LMS algorithm, and it is necessary to use an appropriate value for the performance of the
(LMS) algorithm .from the fig (2), we can notice that the algorithm (LMS) at a lower value of $\mu$, the algorithm converges slowly, then the cost function become high and gives high error rate. but for a large value of $\mu$ the algorithm converges faster and gives a lower cost function with a lower error rate.

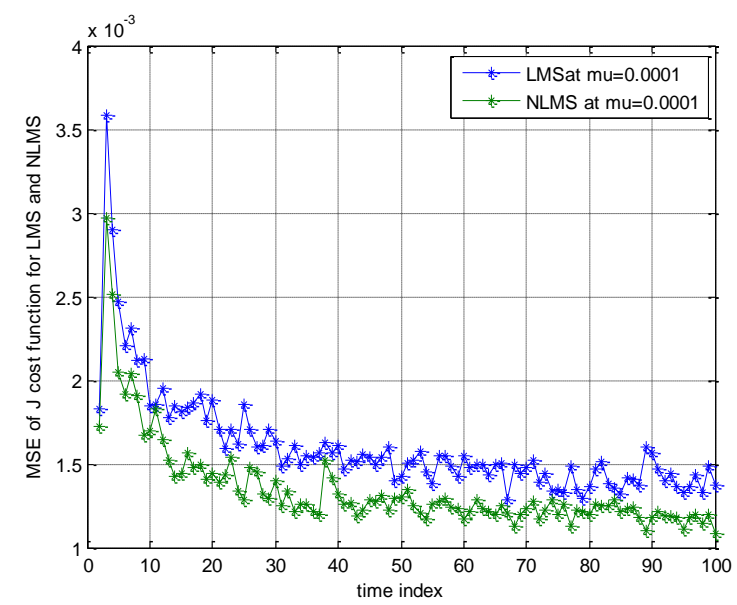

Fig. 3. Compare performance between (LMS) and (NLMS) algorithm.

During complete execution, one of the main disadvantages of the LMS algorithm is having a fixed step size parameter. This calls for an understanding of the input signal statistics prior to the adaptive filtering process starts.

An extension of the (LMS) algorithm that bypasses this problem by choosing a different step size value $\mu$ for each iteration of the algorithm is the normalized least mean square (NLMS). The increase in step size leads to faster convergence, from the figure it can be seen that the algorithm (NLMS) converges faster than the algorithm (LMS) at the same step size parameter, and thus the value of the MSE after converges will be low and higher stability can be obtained.

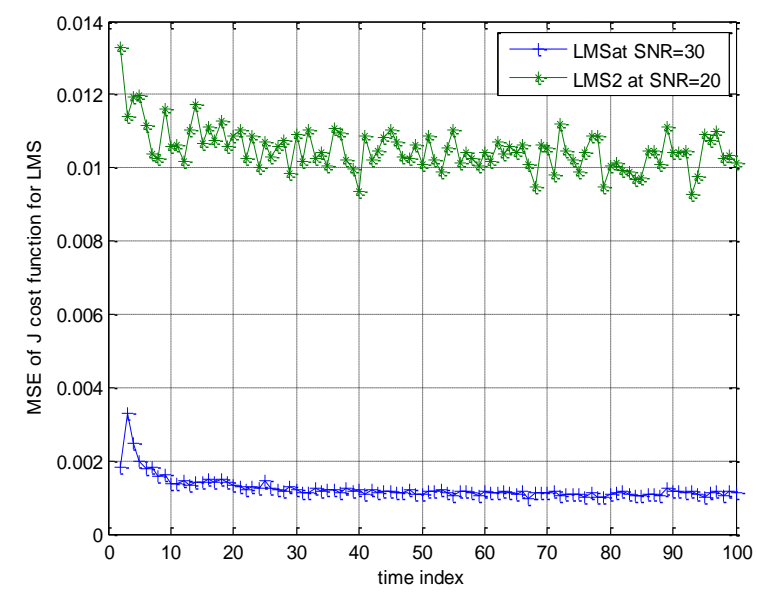


Fig. 4. compares between the cost function of the (LMS) algorithm at different values for SNR.

To tracking the best beam we can use the algorithm that has the least error, In Fig. 4, we can note that the tracking algorithm (LMS) have a cost function at $\mathrm{SNR}=30$ less than the cost function at $\mathrm{SNR}=20$, this means that the (LMS) algorithm at high SNR has abetter performance with lower error and gives higher stability.

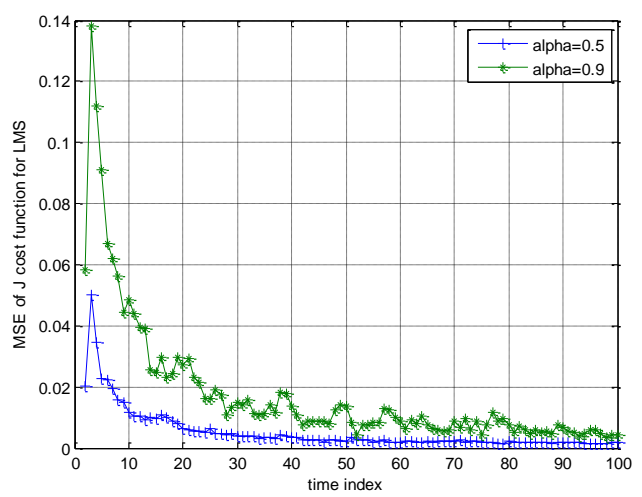

Fig.5. comparison between cost function of the LMS algorithm for different value of $\alpha$.

The attenuation factor has an effect on improving the algorithm, as increasing the attenuation factor means weakening the signal, and thus the improvement in the algorithm is less, as in fig (5)

\section{CONCLUSION}

In this paper, channel tracking and adaptive beam formation were studied using adaptive algorithrithms (LMS, NLMS), the algorithms were derived under a non-linear control model. The results showed that the algorithm (LMS) is one of the easiest and simplest types of algorithms and needs large step size values to converge faster and thus obtain a stable system. The algorithm (NLMS) is considered a special implementation of the algorithm (LMS) when choosing a normal step size leads to a more stable and convergent adaptive algorithm.

\section{REFERENCES}

[1] A., Ali, and Mohamed Deriche. "LMS for Truncate CSI Feedback in Massive MIMO." 2019 International Conference on Computing, Electronics \& Communications Engineering (iCCECE). IEEE, 2019.

[2] X., Xiaoxuan, et al. "Low Complexity Angular-Domain Detection for the Uplink of Multi-User mmWave Massive MIMO Systems." Electronics 9.5 (2020): 795
[3] S., Shu, and Theodore S. Rappaport. "Millimeter wave MIMO channel estimation based on adaptive

[4] C., Hyeonjin, et al. "Adaptive beamwidth control for mmWave beam tracking." IEEE Communications Letters (2020).

[5] L., Jaechan, Hyung-Min Park, and Daehyoung Hong. "Beam Tracking Under Highly Nonlinear Mobile Millimeter-Wave Channel." IEEE Communications Letters 23.3 (2019): 450-453.

[6] P., Saurabh R., and Bhalchandra B. Godbole. "Optimization of LMS Algorithm for System Identification." arXiv preprint arXiv:1706.00897 (2017).

[7] R., Md Faisal, and AHM Asadul Huq. "Comparison between LMS \& NLMS Algorithms in Adaptive Noise Cancellation for Speech Enhancement."2015.

[8] P., Dhaval N., B. J. Makwana, and P. B. Parmar. "Comparative analysis of adaptive beamforming algorithm LMS, SMI and RLS for ULA smart antenna." 2016 International Conference on Communication and Signal Processing (ICCSP). IEEE, 2016.

[9] V., Vutha, Haris Vikalo, and Robert W. Heath. "Beam tracking for mobile millimeter wave communication systems." 2016 IEEE Global Conference on Signal and Information Processing (GlobalSIP). IEEE, 2016.

[10] Y., Yavuz, and Ismail Güvenç. "Lowcomplexity adaptive beam and channel tracking for mobile mmWave communications." 2018 52nd Asilomar Conference on Signals, Systems, and Computers. IEEE, 2018.

[11] J., Anila. "Simulation of an Adaptive Digital Beamformer using Matlab." Simulation 2.12 (2016)..

[12] G., Sajjad Ahmed, and Muhammad Farhan Sohail. "System identification using LMS, NLMS and RLS." 2013 IEEE Student Conference on Research and Developement. IEEE, 2013. 


\section{قناة تتبع الثعاع لنظام اتصالات الموجات المليمترية باستخدام خوارزمية المربع الاقل متوسطا}

** هر هاد عز الاين محمود

farhad.m@uomosul.edu.iq
* بان عزيز عاصي

ban.enp1@student.uomosul.edu.iq

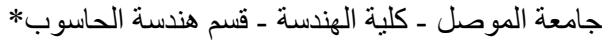

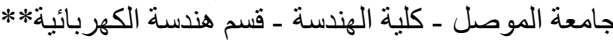

(ألملنص

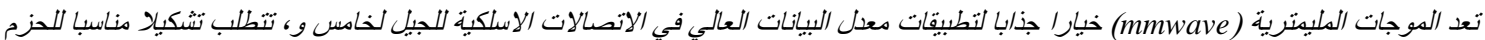

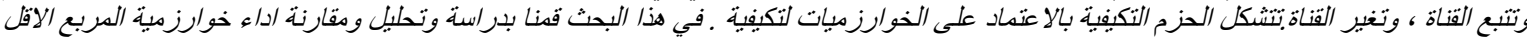

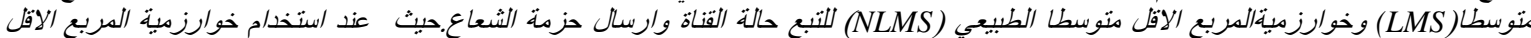

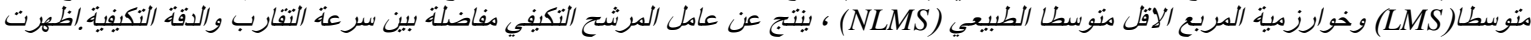

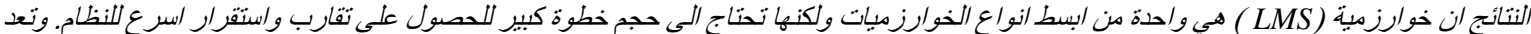

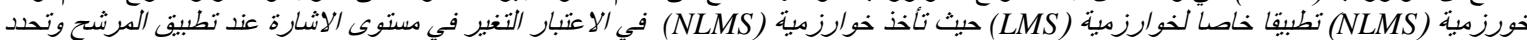

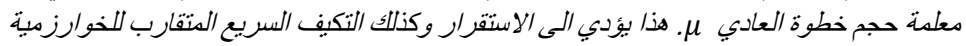

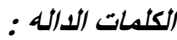

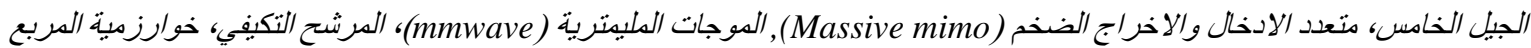
الاقل متوسطا (LMS)، خوارزمبية المربع الاقل متوسطا الطبيعي (NLMS). 